Supporting Information for

\title{
Cu-catalyzed Atom Transfer Radical Polymerization in the Presence of Liquid Metal Micro/nanodroplets
}

Qiangbing Wei ${ }^{\S \dagger}$, Mingkang Sun ${ }^{\S}$, Francesca Lorandi, Rongguan Yin, Jiajun Yan, Tong Liu, Tomasz Kowalewski, Krzysztof Matyjaszewski*

Department of Chemistry, Carnegie Mellon University, Pittsburgh, Pennsylvania 15213, United States.

*Corresponding author: matyjaszewski@,cmu.edu

${ }^{\S}$ These authors contributed equally. 


\section{Contents}

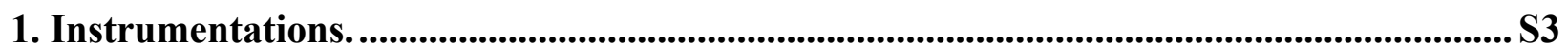

2. Additional experimental details of ATRP in the presence of EGaIn .............................S3

3. Procedure of ATRP in the presence of EGaIn-g-PAA-b-PMMA................................... S4

4. Calculation of rate coefficients of key processes of ATRP in the presence of EGaIn. ..... S4

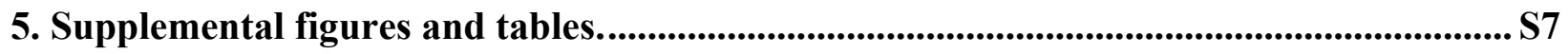

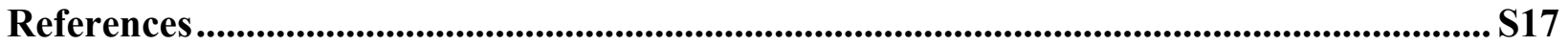




\section{Instrumentations.}

${ }^{1} \mathrm{H}$ NMR was conducted on a Bruker $500 \mathrm{MHz}$ AVANCE III NMR spectrometer. THF GPC was equipped with a Waters 515 HPLC pump and a Waters 2410 refractive index detector, with PSS SDV analytical columns ( $5 \mu \mathrm{m}$ particle size, column temperature was set to $\left.35^{\circ} \mathrm{C}\right)$ and HPLC grade THF as eluent. Molecular weight was calculated using PSS WinGPC software based on a conventional calibration using a series of linear PMMA standards (toluene as the smallmolecule internal standard). Agilent InfinityLab EasiVial standards were used for calibration. Dynamic light scattering (DLS) was performed on a Malvern Zetasizer Nano system. For size measurements of EGaIn droplets after polymerization, the droplets were separated from the polymerization mixture via centrifugation and underwent several circles of washing (with acetone) to remove residue polymer and monomer. Transmission electron microscopy (TEM) was performed on a JEOL JEM-2000EX TEM using a Gatan ES500W Erlangshen camera, with an accelerating voltage of $200 \mathrm{kV}$ and working current of $112 \mu \mathrm{A}$. TEM samples were prepared by drop-casting the diluted DMF dispersion of EGaIn onto the surface of $\mathrm{Cu}$ grids and dried under ambient conditions overnight. Scanning electron microscope (SEM) was conducted on a Quanta 600 FEG instrument. SEM samples were prepared by depositing EGaIn dispersion onto a Si wafer. Sonication was performed using a Branson M1800 ultrasound cleaner with a water bath at $25{ }^{\circ} \mathrm{C}$ and default intensity. Linear sweep voltammetries were measured by an Autolab PGSTAT302N potentiostat/galvanostat (Metrohm) run by a PC with NOVA 2.0 software.

\section{Additional experimental details of ATRP in the presence of EGaIn}

The following experiments were carried out according to the procedure enclosed in the main manuscript. For reactions using $50 \mathrm{mg}$ and $25 \mathrm{mg}$ EGaIn, $2.5 \mathrm{~mL}$ and $1.25 \mathrm{~mL}$ of the original EGaIn dispersion $(20 \mathrm{mg} / \mathrm{mL})$ were charged in the Schlenk flask and were diluted with $2.5 \mathrm{~mL}$ 
and $3.75 \mathrm{~mL}$ DMF, respectively. For reactions using $200 \mathrm{mg}$, the $10 \mathrm{~mL}$ dispersion was placed still on a benchtop and form sedimentation until $5 \mathrm{~mL}$ of pure DMF can be easily withdrawn from the supernatant.

For ATRP with different monomers (all entries in Table 2), $5 \mathrm{~mL}$ EGaIn dispersion containing $100 \mathrm{mg}$ EGaIn was used. When using butyl acrylate as the monomer, anisole was used to disperse EGaIn.

For ATRP in the presence of EGaIn without deoxygenation, a magnetic stir bar, $5 \mathrm{~mL} 20$ $\mathrm{mg} / \mathrm{mL}$ pre-dispersed EGaIn, $1.81 \mathrm{~mL}$ MA, $1.79 \mathrm{mg} \mathrm{CuBr} 2,9.22 \mathrm{mg}$ Мe $6 \mathrm{TREN}$ and $9.78 \mu \mathrm{L} \mathrm{EBiB}$ was added to a $7 \mathrm{~mL}$ glass vial. The vial was then capped, placed on a magnetic stir plate and was then reacted for $8 \mathrm{~h}$.

\section{Procedure of ATRP in the presence of EGaIn-g-PAA-b-PMMA.}

The synthesis of block copolymer surfactant, PAA65-b-PMMA45, was synthesized using previously reported methods. ${ }^{1}$ In a typical experiment, $200 \mathrm{mg}$ EGaIn and $10 \mathrm{mg}$ copolymer were weighed and charged in a glass vial with $10 \mathrm{~mL}$ DMF. Then, the mixture was sonicated, and ATRP was carried out using the same procedure as reactions using bare EGaIn.

\section{Calculation of rate coefficients of key processes of ATRP in the presence of EGaIn.}

To quantify the contribution from EGaIn-based activation and $\mathrm{Cu}^{\mathrm{I}}$-based activation, the rate coefficient for EGaIn-based activation of alkyl bromide $\left(k_{\mathrm{a} 0}\right)$ and for EGaIn-based reduction $\left(k_{\mathrm{RA}}\right)$ were calculated using eq. $1-2$ in the manuscript. ${ }^{2}$ Note that the $k_{\mathrm{RA}}$ in this context is equivalent to $0.5 \times k_{\mathrm{RA}}$ in ref [2], since two units of $\mathrm{Cu}^{\mathrm{I}}$ is generated when using $\mathrm{Cu}(0)$ as the reducing agent. $R_{\mathrm{p} \text {,with Cu}}$ and $R_{\mathrm{p} \text {,without Cu}}$ corresponded to the rate of propagation when $\mathrm{CuBr}_{2}$

was present and absent, respectively. $k_{\mathrm{p}}$ and $k_{\mathrm{t}}$ referred to rate coefficients for propagation and 
termination of MA, respectively. $[\mathrm{M}],[\mathrm{RX}]$ and $[\mathrm{Cu} / \mathrm{LI}]$ referred to concentration of monomer, alkyl bromide and $\mathrm{Cu}$ species, and $(S / V)$ was the ratio of the total surface area of EGaIn to the volume of reaction.

The rate of activation by EGaIn $\left(R_{\mathrm{a} 0}\right)$ and rate of reduction by EGaIn $\left(R_{\mathrm{RA}}\right)$ were calculated using eq. S1-S2:

$$
\begin{aligned}
& R_{\mathrm{a} 0}=k_{\mathrm{a} 0}(S / V)[\mathrm{RX}] \\
& R_{\mathrm{RA}}=k_{R A}(S / V)\left[\mathrm{Cu}^{\mathrm{II}} / \mathrm{L}\right]
\end{aligned}
$$

Furthermore, by using the ATRP equilibrium constant (KATRP) and the total concentration of $\mathrm{Cu}$ species, the concentration of $\mathrm{Cu}^{\mathrm{I}}$ complex $\left(\left[\mathrm{Cu}^{\mathrm{I}} / \mathrm{L}\right]\right)$ was calculated, which was subsequently applied to eq. 3 in the manuscript to calculate the rate of activation by $\mathrm{Cu}^{\mathrm{I}}\left(R_{\mathrm{a} 1}\right):^{3}$

The following rate coefficients were obtained from literature, either directly or with scaling:

\begin{tabular}{|c|c|c|}
\hline Coefficients & Referenced value & Reference and note \\
\hline$k_{\mathrm{p}}$ & $15600 \mathrm{M}^{-1} \mathrm{~s}^{-1}$ & 4 \\
\hline$k_{\mathrm{t}}$ & $1 \times 10^{8} \mathrm{M}^{-1} \mathrm{~s}^{-1}$ & \\
\hline$K_{\text {ATRP }}$ & $7.1 \times 10^{-6}$ & ${ }^{5-7}$ (scaled) \\
\hline$k_{\mathrm{a} 1}$ & $108 \mathrm{M}^{-1} \mathrm{~s}^{-1}$ & ${ }^{6}$ (scaled) \\
\hline
\end{tabular}

$k_{\mathrm{a} 1}$ correspond to rate coefficients in the presence of methyl 2-bromopropionate $(\mathrm{MBrP})$, mimicking the structure of propagating chain-end using methyl acrylate as the monomer. The adjustments of $K_{\mathrm{ATRP}}$ and $k_{\mathrm{a} 1}$ were performed based on the following methods:

- $\quad$ Scaling for $K_{\mathrm{ATRP}}$ :

According to ref [5], for ATRP of MA with $\mathrm{CuBr} / \mathrm{Me}_{6} \mathrm{TREN}$ in 75 vol\% of DMSO $K_{\text {ATRP }}$ $=3.04 \times 10^{-5}$, as calculated from the following equation: 


$$
\ln \left(K_{A T R P}\right)=-17.0+8.8 f_{S}(D M S O)
$$

Where, $f_{S}(D M S O)$ is the volume fraction of DMSO.

Then, according to ref [7], the $K_{\text {ATRP }}$ in pure DMF has a value of $17.6 \%$ of the $K_{\text {ATRP }}$ in pure DMSO. ${ }^{7}$ Thus, at $75 \%$ vol. DMF, the scaled value should be $K_{\text {ATRP }}=7.1 \times 10^{-6}$.

- Scaling for $k_{\mathrm{a} 1}$ :

Based on ref [6], the activation rate coefficient of $\mathrm{Cu}^{\mathrm{I}} / \mathrm{Me}_{6} \mathrm{TREN}$ with $\mathrm{MBrP}$ in pure acetonitrile is $k_{\mathrm{a} 1}=28 \mathrm{M}^{-1} \mathrm{~s}^{-1}$. According to the solvent effects, ${ }^{8}$ the activation rate coefficient in pure DMF should be 4.3 times higher than that in acetonitrile.

Then, considering the influence of monomer, in a typical ATRP in the presence of EGaIn, for MA content of $24 \mathrm{vol} \%$ in the reaction mixture. Based on ref [8], when using $20 \mathrm{vol} \%$ MA to dilute DMF, the value of $k_{\mathrm{a} 1}$ should correspond to $89 \%$ of the rate coefficient in pure $\mathrm{DMF}^{8}$. Thus, the $k_{\mathrm{a} 1}$ value should be:

$$
k_{\mathrm{a} 1}=28 \mathrm{M}^{-1} \sec ^{-1} \times 4.3 \times 0.89=108 \mathrm{M}^{-1} \mathrm{~s}^{-1} .
$$

- Calculation of surface area:

An average diameter of EGaIn $d=900 \mathrm{~nm}$ based on DLS measurements was used to calculate the specific surface area. The total surface area was calculated using eq. S3:

$$
S A_{\text {total }}=\frac{V_{\text {total }}}{V_{\text {each }}} \times S A_{\text {each }}
$$

Where, $S A_{\text {each }}$ is the surface area of each EGaIn droplet $\left(S A_{\text {each }}=4 \pi \times(0.5 \times d)^{2}\right.$, where $d$ is the average diameter of EGaIn droplets)), $V_{\text {each }}$ is the volume of each EGaIn droplet $\left(V_{\text {each }}=\frac{4 \pi \times(0.5 \times d)^{2}}{3}\right)$, and $V_{\text {total }}$ is the total volume of EGaIn, which is the ratio of the mass to the density of bulk EGaIn $\left(V_{\text {total }}=\frac{M_{E G a I n}}{\rho_{E G a I n}}\right)$. 
Based on these calculations, calculated parameters were listed in Table S1.

\section{Supplemental figures and tables.}

Table S1. Kinetic parameters calculated for conditions corresponding to entry 2 in Table 1:

\begin{tabular}{|c|c|c|}
\hline Parameter & Calculated value & Definition \\
\hline$R_{\mathrm{p}}$ & $3.30 \times 10^{-4} \mathrm{M} \mathrm{s}^{-1}$ & Rate of propagation \\
\hline $\begin{array}{c}R_{\mathrm{p}}(\text { control, } \\
\text { no } \mathrm{Cu})\end{array}$ & $2.45 \times 10^{-5} \mathrm{M} \mathrm{s}^{-1}$ & Rate of propagation, with no $\mathrm{CuBr}_{2}$ \\
\hline$S / V$ & $156 \mathrm{~cm}^{-1}(d=900 \mathrm{~nm})$ & Ratio of EGaIn surface area to solution volume \\
\hline$k_{\mathrm{a} 0}$ & $1.9 \times 10^{-11} \mathrm{~cm} \mathrm{~s}^{-1}$ & Rate coefficient of activation by $\mathrm{EGaIn}$ \\
\hline$k_{\mathrm{RA}}$ & $2.8 \times 10^{-8} \mathrm{~cm} \mathrm{~s}^{-1}$ & Rate coefficient of reduction of $\mathrm{Cu}$ \\
\hline$\left[\mathrm{R}^{\bullet}\right]$ & $7.2 \times 10^{-9} \mathrm{M}^{\mathrm{II}}$ & Concentration of propagating radical \\
\hline$R_{\mathrm{a} 0}$ & $2.9 \times 10^{-11} \mathrm{M} \mathrm{s}^{-1}$ & Rate of activation by $\mathrm{EGaIn}$ \\
\hline$R_{\mathrm{RA}}$ & $5.2 \times 10^{-9} \mathrm{M} \mathrm{s}^{-1}$ & Rate of Cu $\mathrm{u}^{\mathrm{II}}$ reduction \\
\hline$[\mathrm{Cu} / \mathrm{L}]$ & $0.08 \mathrm{mM}^{\mathrm{I}}$ & Concentration of $\mathrm{Cu} / \mathrm{L}$ \\
\hline$[\mathrm{Br}-\mathrm{Cu} / \mathrm{II}]$ & $1.09 \mathrm{mM}^{\mathrm{I}}$ & Concentration of $\mathrm{Br}-\mathrm{Cu}{ }^{\mathrm{II}} / \mathrm{L}$ \\
\hline$R_{\mathrm{a} 1}$ & $8 \times 10^{-5} \mathrm{M} \mathrm{s}^{-1}$ & Rate of activation by $\mathrm{Cu} / \mathrm{L}$ \\
\hline
\end{tabular}



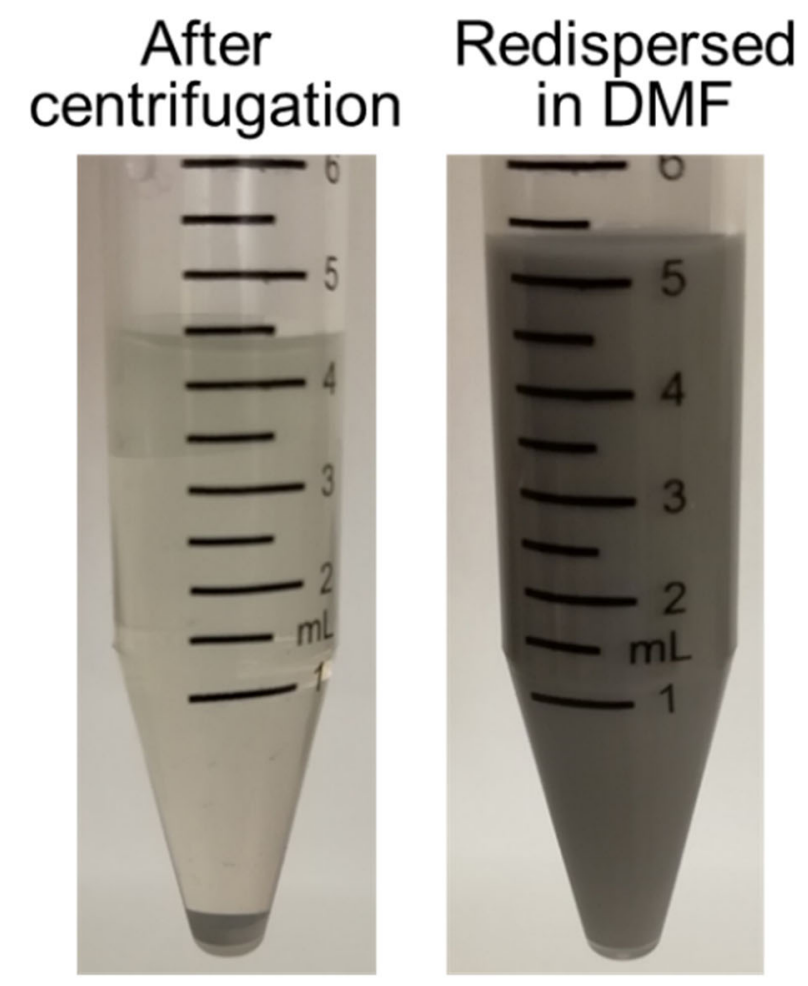

Regenerated
by acidification
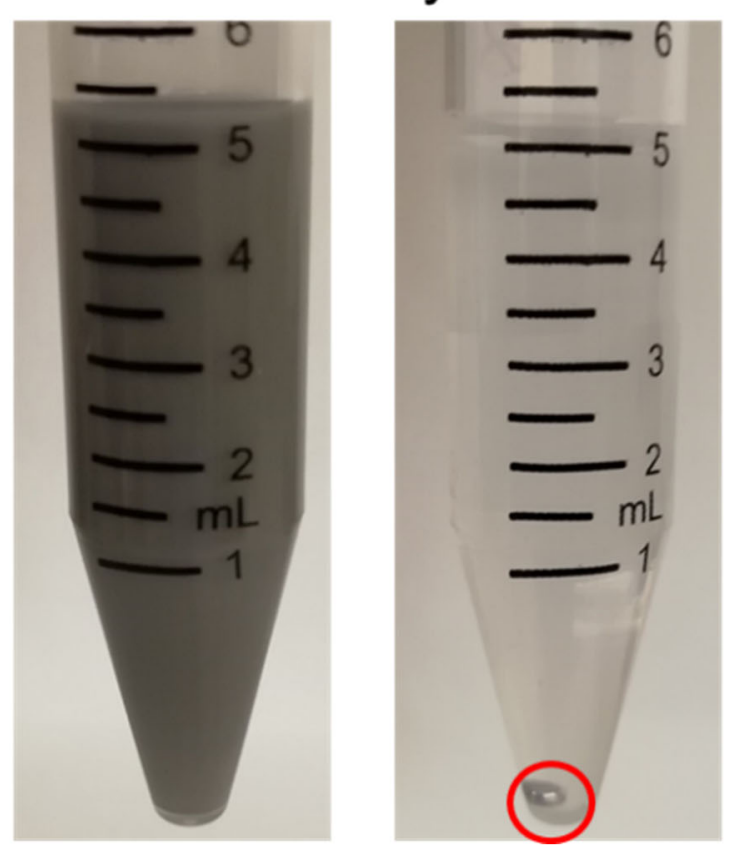

Figure S1. Images of a polymerization mixture after polymerization and centrifugation (left), redispersed in DMF (middle) and after adding acid $(\sim 0.05 \mathrm{~mL} 6 \mathrm{M} \mathrm{HCl})$ to regenerate EGaIn. 

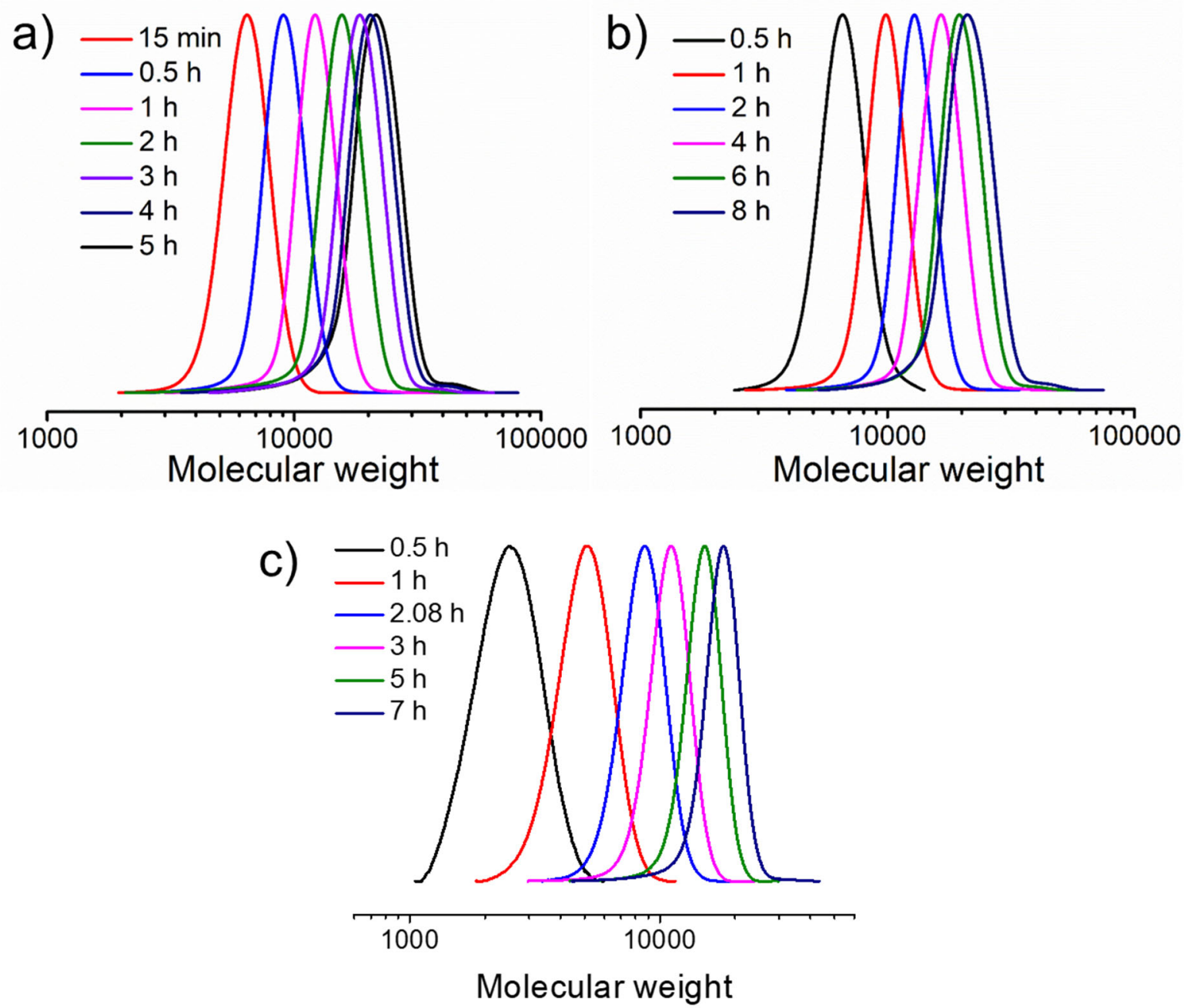

Figure S2. GPC traces of samples taken at different times during the reactions corresponding to a) entry 1, b) entry 3 and c) entry 4 condition of Table 1 (THF as eluent). 


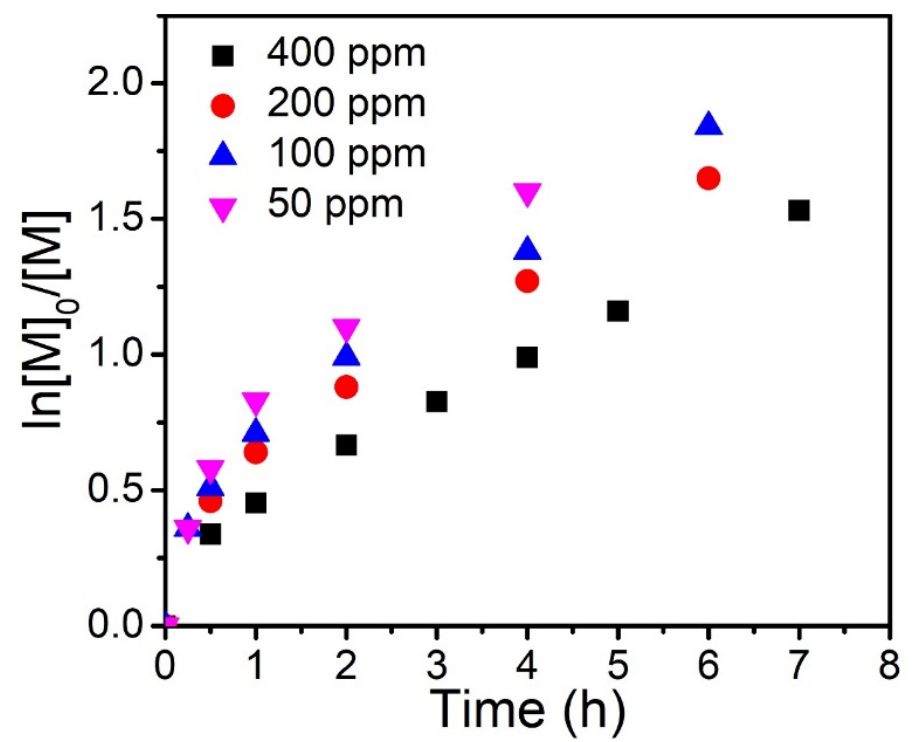

Figure S3. Semilogarithmic kinetic plots for entry 2 (labeled as 400 ppm) and entries 5-7 (200, 100 and $50 \mathrm{ppm})$ in Table 1.

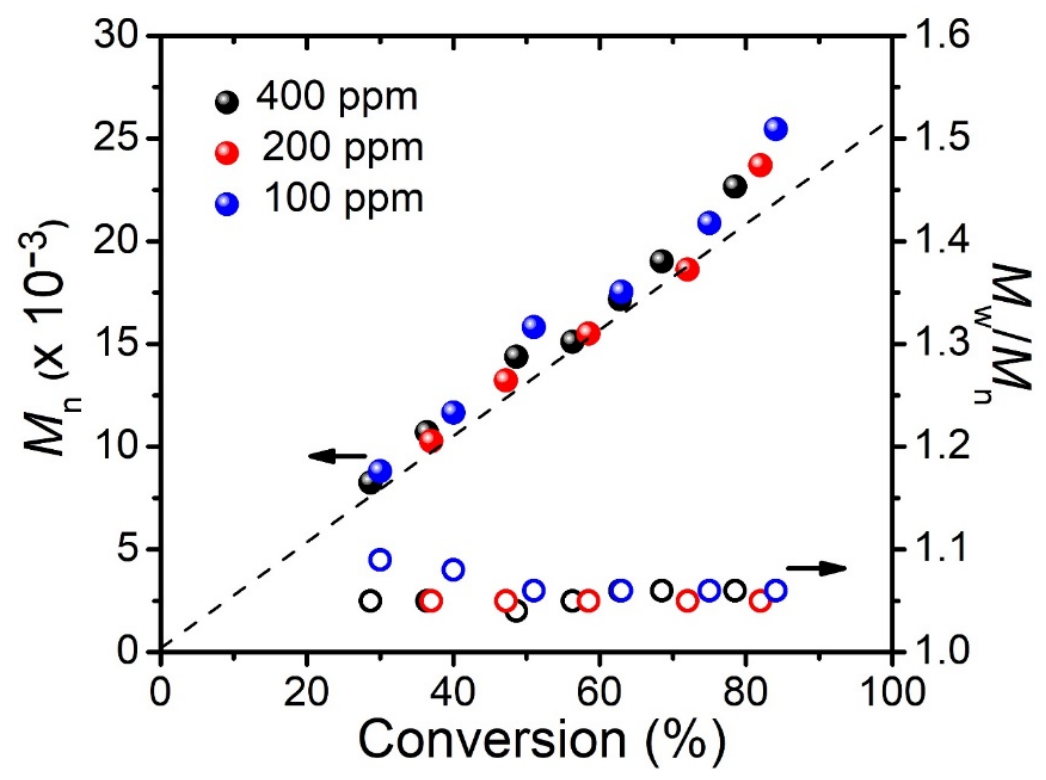

Figure S4. Plot of the MW measured by GPC ( $M_{\mathrm{n}, \mathrm{GPC}}$, solid dots), dispersity ( $Ð$, open dots) vs. the monomer conversion for reactions of entry $2(400 \mathrm{ppm})$ and entries $5(200 \mathrm{ppm})$ to $6(100 \mathrm{ppm})$ in Table 1. 


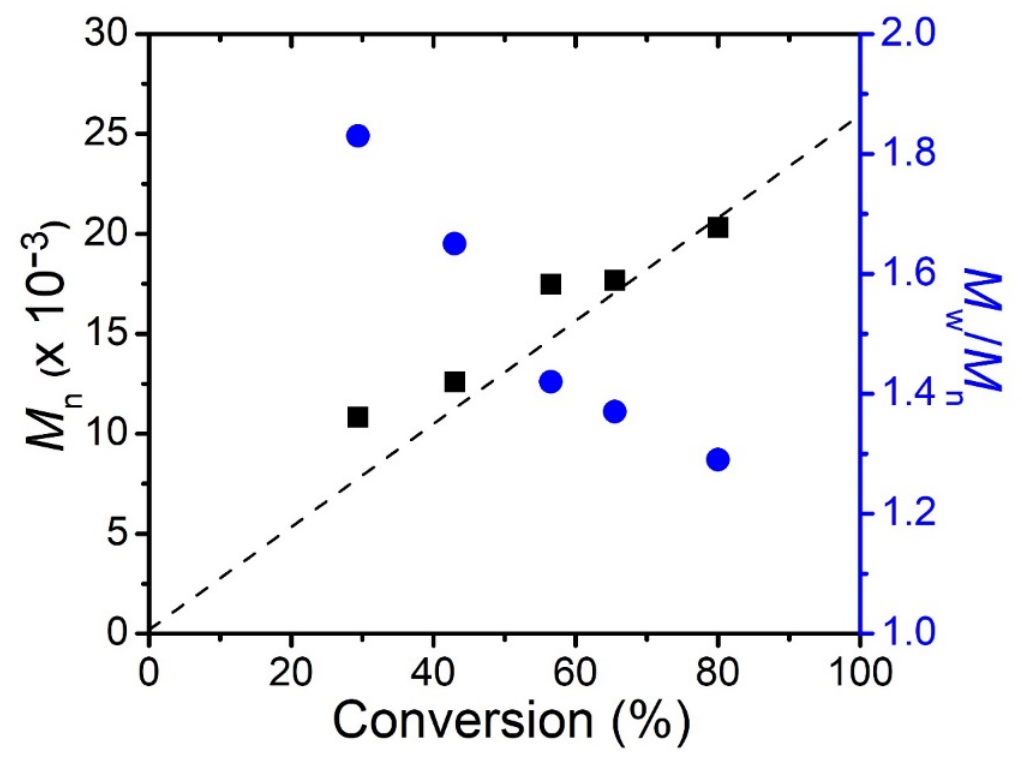

Figure S5. Plot of the MW measured by GPC ( $M_{\mathrm{n}, \mathrm{GPC}}$, black squares), dispersity $(\nexists$, blue dots) and the monomer conversion for reactions of entry 7 in Table 1.

Table S2. Conditions and results of additional ATRP experiments in the presence of EGaIn of MA with different $\mathrm{DP}_{\text {target }}$ and ATRP ligand. $400 \mathrm{ppm} \mathrm{CuBr}_{2}$ was used for all entries.

\begin{tabular}{|cccccccccc|}
\hline Entry & $\begin{array}{c}\text { EGaIn } \\
(\mathbf{m g})\end{array}$ & Ligand & $\begin{array}{c}\mathbf{C u B r}_{2}: \\
\text { Ligand }\end{array}$ & $\mathbf{D P}_{\text {target }}$ & $\begin{array}{c}\text { Time } \\
(\mathbf{h})\end{array}$ & $\begin{array}{c}\text { Conv } \\
(\mathbf{\%})\end{array}$ & $\boldsymbol{M}_{\mathbf{n}, \text { th }}$ & $\boldsymbol{M}_{\mathbf{n}, \mathrm{app}}$ & $\boldsymbol{M}_{\mathbf{w}} \boldsymbol{M}_{\mathbf{n}}$ \\
\hline 1 & 200 & $\mathrm{Me}_{6}$ TREN & $1: 5$ & 600 & 6 & 66 & 34300 & 35000 & 1.18 \\
\hline 2 & 200 & $\mathrm{Me}_{6}$ TREN & $1: 5$ & 900 & 6 & 65 & 50500 & 45600 & 1.17 \\
\hline 3 & 100 & TPMA & $1: 5$ & 300 & 8 & 73 & 19000 & 23000 & 1.23 \\
\hline 4 & 100 & TPMA & $1: 1$ & 300 & 8 & 44 & 11700 & 13100 & 1.05 \\
\hline 5 & 100 & $\mathrm{Me}_{6}$ TREN & $1: 1$ & 300 & 8 & 78 & 20200 & 21300 & 1.08 \\
\hline
\end{tabular}




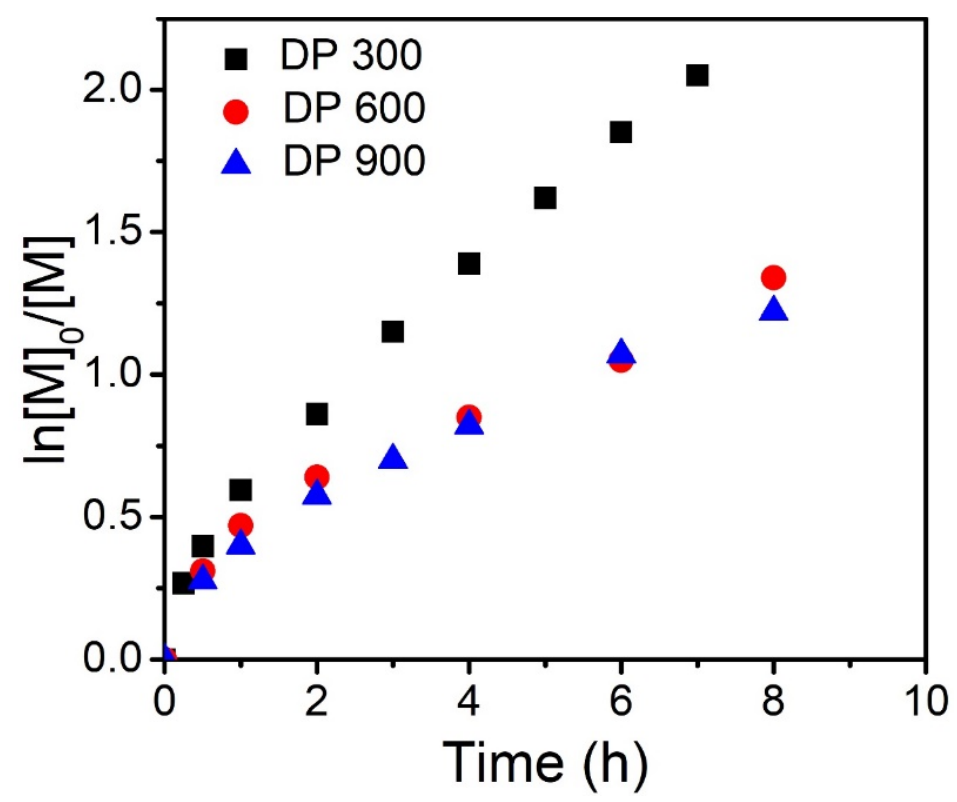

Figure S6. Semilogarithmic kinetic plots for entry 1 in Table 1 (DP 300), entries 1 (DP 600) and 2 (DP 900) in Table S2.

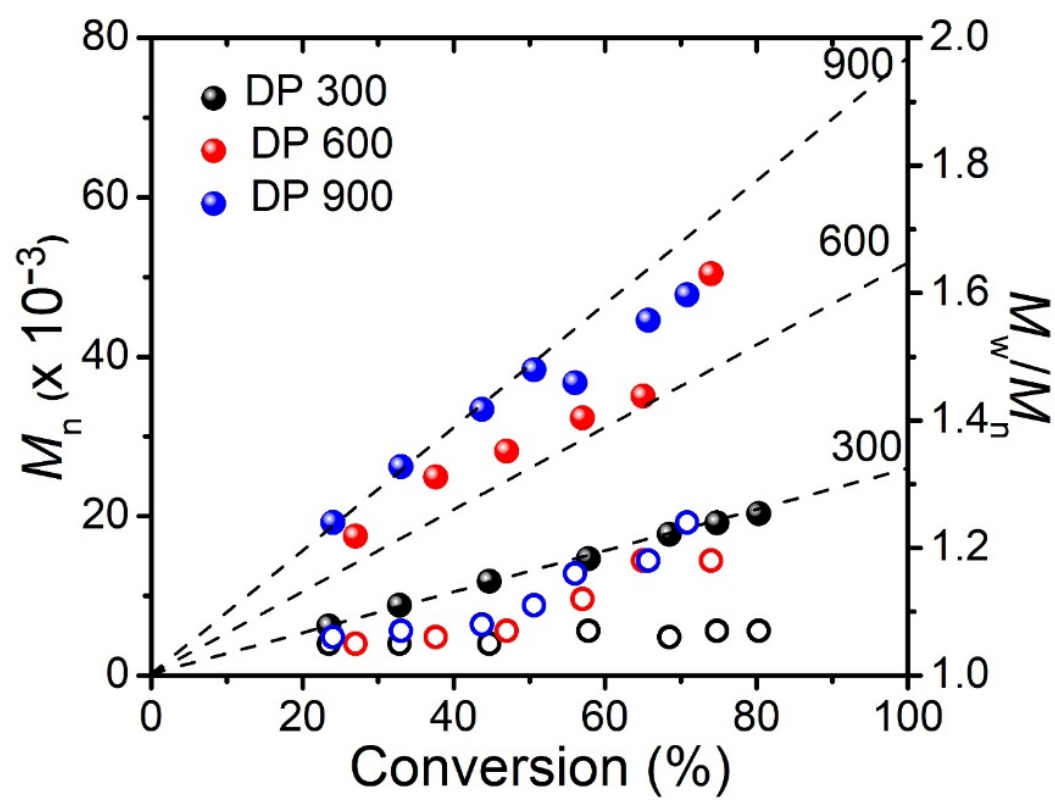

Figure S7. Dependence of the MW measured by GPC ( $M_{\mathrm{n}, \mathrm{GPC}}$, solid dots), dispersity ( $\nexists$, open dots) on the monomer conversion for reactions of entry 1 in Table 1 (DP 300), entries 1 (DP 600) and 2 (DP 900) in Table S2. 

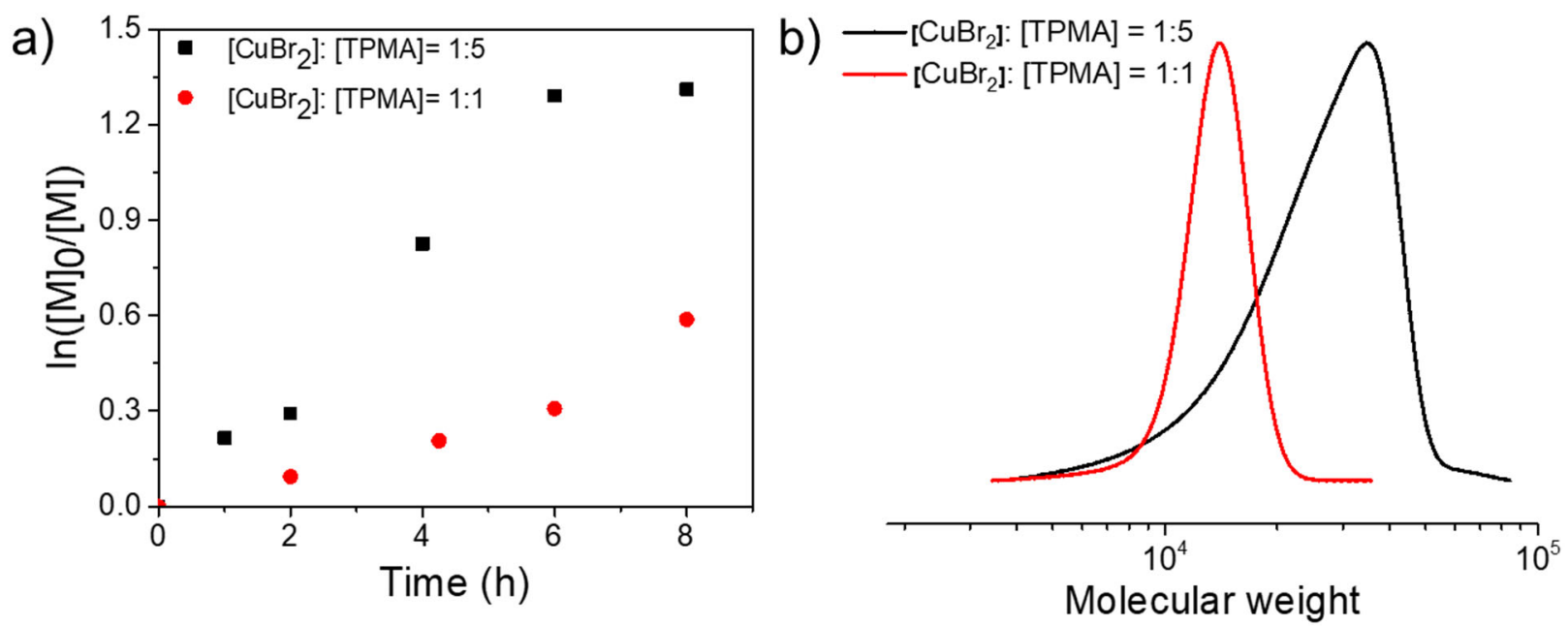

Figure S8. a) Semilogarithmic kinetic plots for entries 3 (black) and 4 (red) of Table S2. b) THF GPC traces (at $8 \mathrm{~h}$ ) corresponding to the reactions in entries 3 (black) and 4 (red) of Table S2.
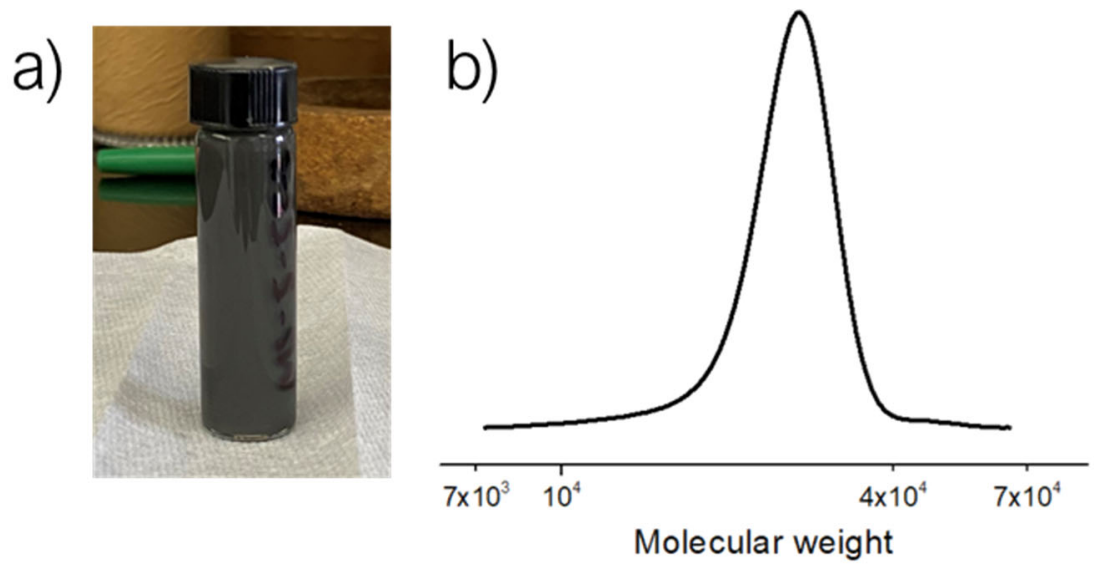

Figure S9. a) An image of the reaction setup of ATRP in the presence of EGaIn without deoxygenation. b) THF GPC trace at $8 \mathrm{~h}$ corresponding to ATRP of MA in the presence of EGaIn without deoxygenation. $M_{\mathrm{n}, \mathrm{GPC}}=2.5 \times 10^{4}, Ð=1.05$. Polymerization molar ratio: $[\mathrm{MA}]_{0}:[\mathrm{EBiB}]_{0}$ : $\left[\mathrm{CuBr}_{2}\right]_{0}:\left[\mathrm{Me}_{6} \mathrm{TREN}_{0}=300: 1: 0.12: 0.6\right.$. 


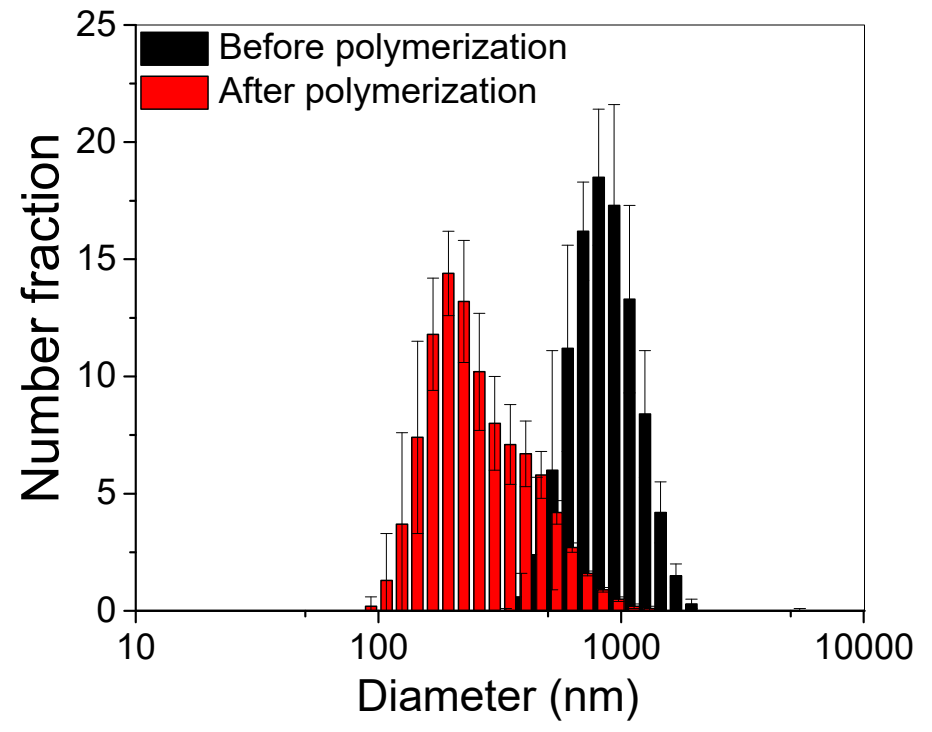

Figure S10. Dynamic light scattering (DLS) number fraction distribution of the particle diameter of EGaIn dispersion before (black) and after (red) polymerization.

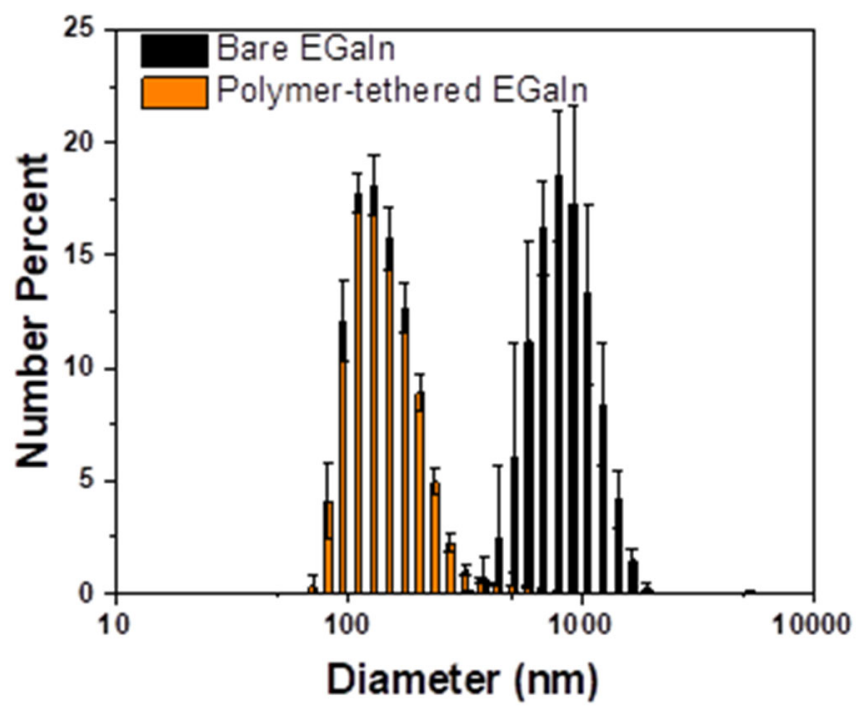

Figure S11. DLS number fraction distribution of particle sizes of bare EGaIn (black) and EGaIn$g$-PAA- $b$-PMMA (orange). Both samples were measured before being used in polymerization. 

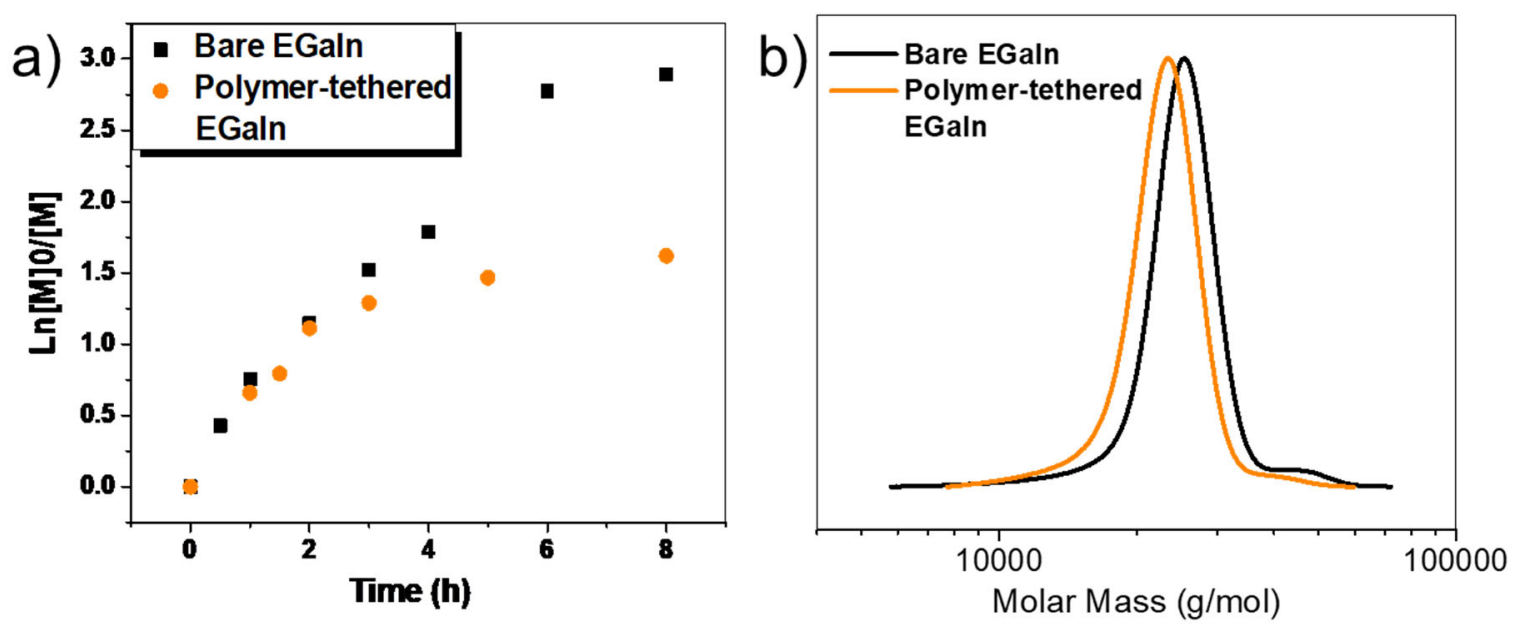

Figure S12. (a) Semilogarithmic kinetic plots and (b) THF GPC traces (at $8 \mathrm{~h}$ ) corresponding to ATRP in the presence of bare EGaIn droplets (black) and EGaIn-g-PAA- $b$-PMMA (orange).

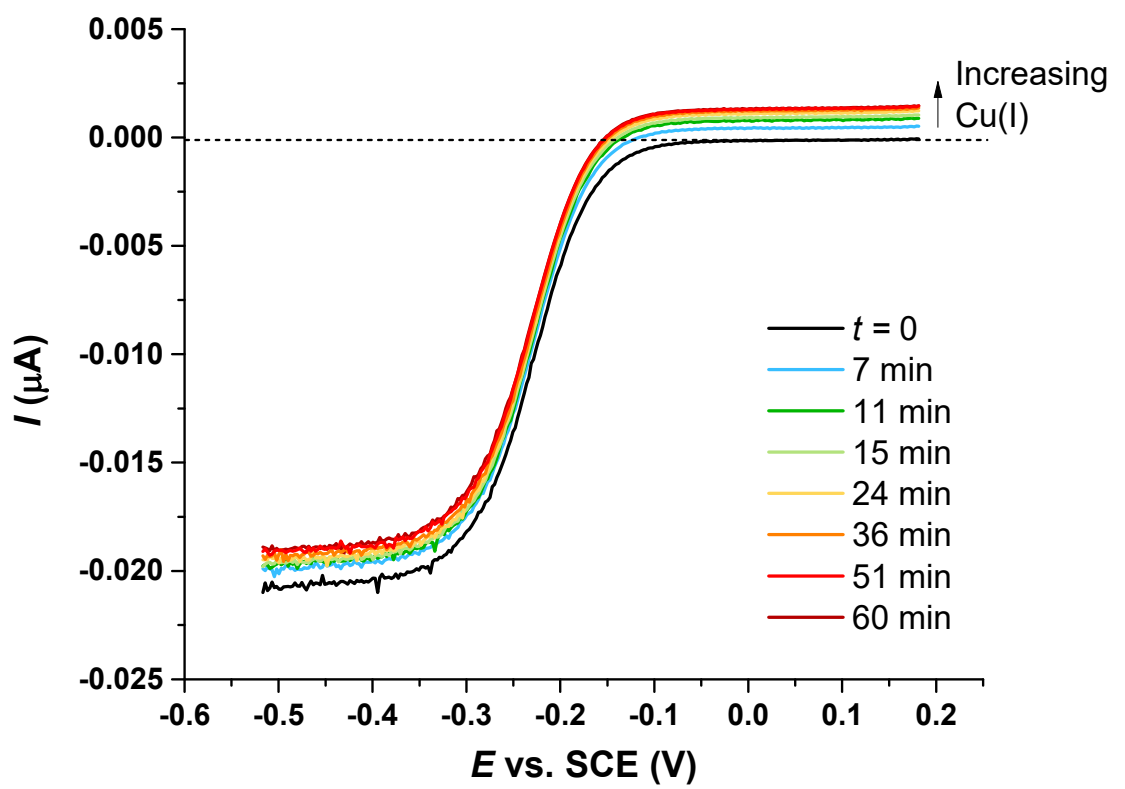

Figure S13. LSV of $0.5 \mathrm{mM} \mathrm{CuBr} / \mathrm{TPMA}$ in DMF with $1 \mathrm{mg} / \mathrm{mL}$ EGaIn- $g$-PAA- $b$-PMMA and $0.1 \mathrm{MEt}_{4} \mathrm{NBF}_{4}$ at $\mathrm{v}=0.01 \mathrm{~V} / \mathrm{s}, \omega=2500 \mathrm{rpm}$, over time. 


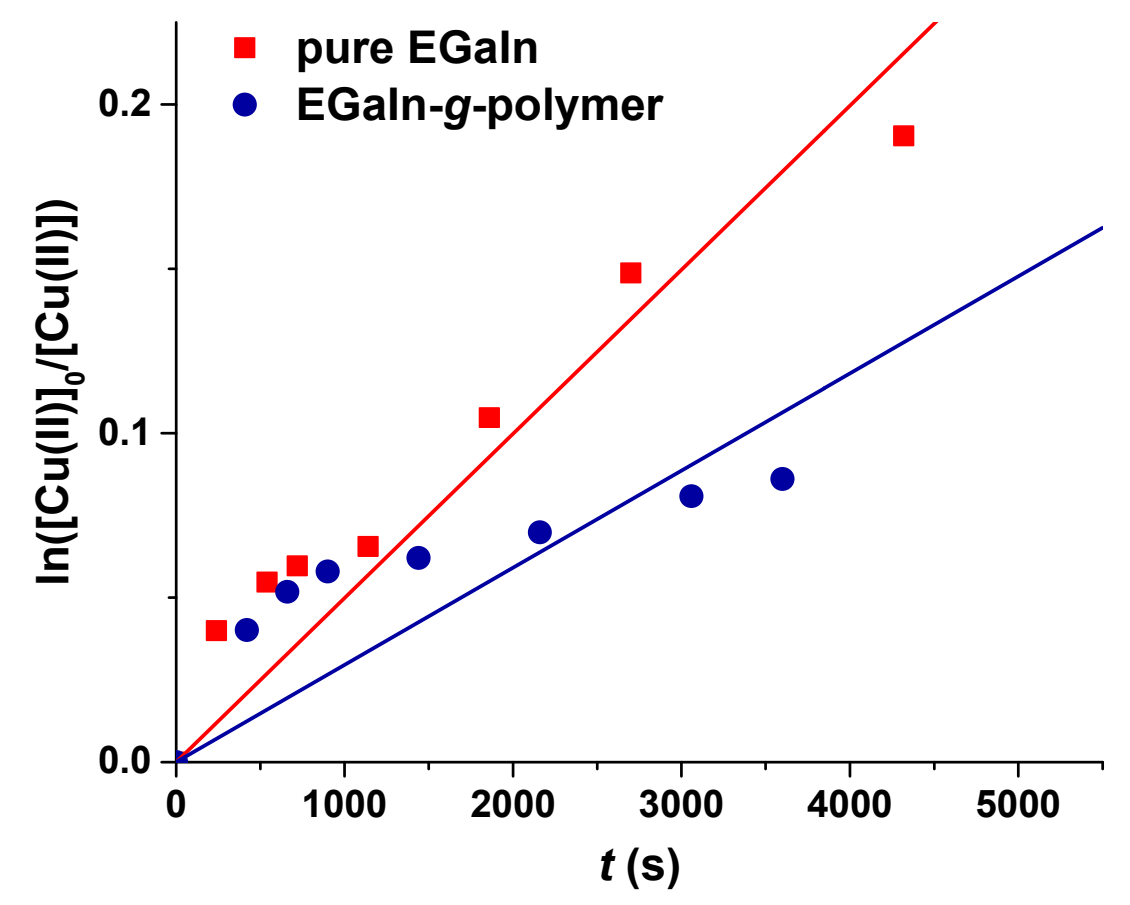

Figure S14. Rate of reduction of $0.5 \mathrm{mM}\left[\mathrm{Br}-\mathrm{Cu}{ }^{\mathrm{II}} / \mathrm{TPMA}\right]^{+}$from pure EGaIn and polymer tethered EGaIn nanodroplets $(1 \mathrm{mg} / \mathrm{mL})$ in DMF $+0.1 \mathrm{M} \mathrm{Et}_{4} \mathrm{NBF}_{4}$, measured by LSV experiments.

Table S3. Rate of reduction of $\left[\mathrm{Br}-\mathrm{Cu}^{\mathrm{II}} / \mathrm{L}\right]^{+}$from pure EGaIn and polymer stabilized EGaIn nanoparticles in DMF, calculated from the LSV experiments, assuming a pseudo-first order kinetics. $k_{\text {red }}$ is the apparent rate constant of reduction calculated from Figure S14. When measured under the same condition, $k_{\mathrm{RA}}=k_{\mathrm{red}} \times(S / V)$.

\begin{tabular}{ccc}
\hline & $\mathbf{L}$ & $\boldsymbol{k}_{\text {red }}\left(\mathbf{1 0}^{\mathbf{5}} \mathbf{s}^{-1}\right)$ \\
\hline Pure EGaIn & Me6TREN & $18.0( \pm 2.0)$ \\
Pure EGaIn & TPMA & $5.83( \pm 0.46)$
\end{tabular}


EGaIn-g-(PMMA-b-PAA) $\quad$ TPMA $\quad 4.20( \pm 0.69)$
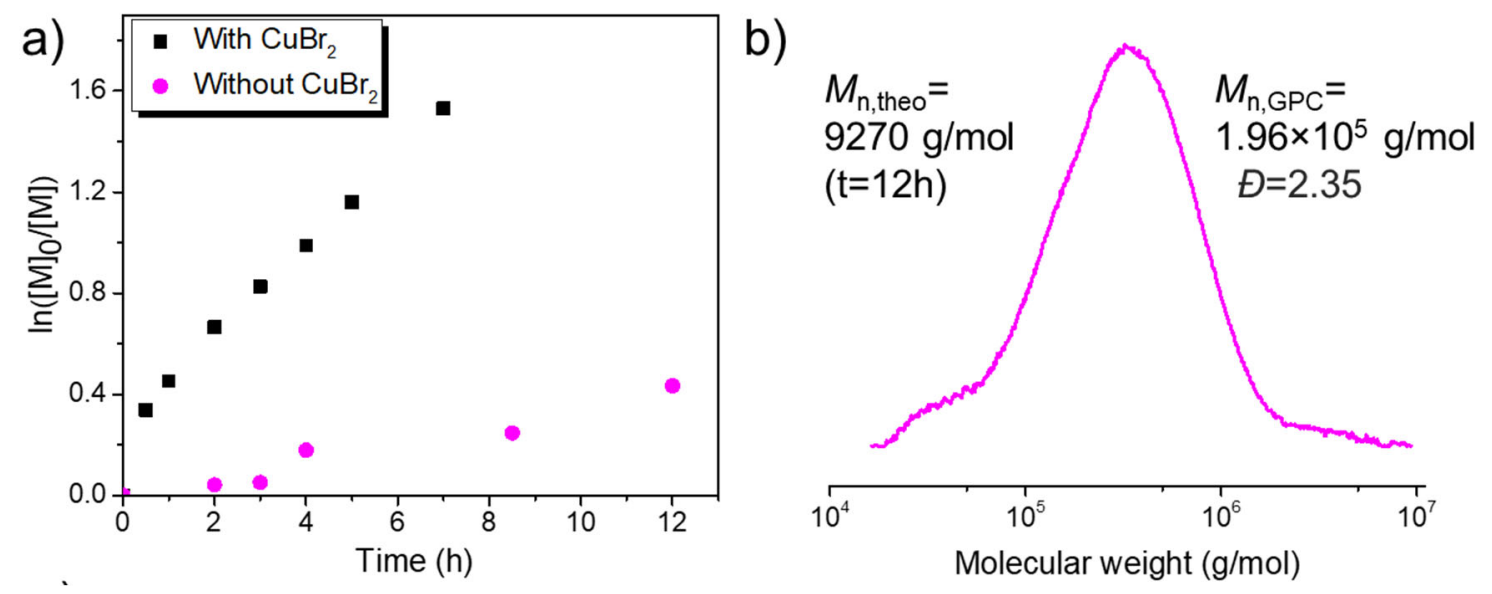

Figure S15. a) Semilogarithmic kinetic plots for entries 1 (black squares) and 8 (magenta dots) in Table 1. b) GPC trace in THF of the polymer obtained from entry 8 of Table 1 at $12 \mathrm{~h}$.

\section{References}

1. Wei, Q. B.; Sun, M. K.; Wang, Z. Y.; Yan, J. J.; Yuan, R.; Liu, T.; Majidi, C.; Matyjaszewski, K., Surface Engineering of Liquid Metal Nanodroplets by Attachable Diblock Copolymers. ACS Nano 2020, 14, 9884-9893.

2. Krys, P.; Ribelli, T. G.; Matyjaszewski, K.; Gennaro, A., Relation between Overall Rate of ATRP and Rates of Activation of Dormant Species. Macromolecules 2016, 49, 2467-2476.

3. Konkolewicz, D.; Krys, P.; Gois, J. R.; Mendonca, P. V.; Zhong, M. J.; Wang, Y.; Gennaro, A.; Isse, A. A.; Fantin, M.; Matyjaszewski, K., Aqueous RDRP in the Presence of Cu-0: The Exceptional Activity of Cu-I Confirms the SARA ATRP Mechanism. Macromolecules 2014, 47, 560570.

4. Zhong, M. J.; Wang, Y.; Krys, P.; Konkolewicz, D.; Matyjaszewski, K., ReversibleDeactivation Radical Polymerization in the Presence of Metallic Copper. Kinetic Simulation. Macromolecules 2013, 46, 3816-3827. 
5. Wang, Y.; Kwak, Y.; Buback, J.; Buback, M.; Matyjaszewski, K., Determination of ATRP Equilibrium Constants under Polymerization Conditions. ACS Macro Lett. 2012, 1, 1367-1370.

6. Tang, W.; Kwak, Y.; Braunecker, W.; Tsarevsky, N. V.; Coote, M. L.; Matyjaszewski, K., Understanding atom transfer radical polymerization: Effect of ligand and initiator structures on the equilibrium constants. J. Am. Chem. Soc. 2008, 130, 10702-10713.

7. Braunecker, W. A.; Tsarevsky, N. V.; Gennaro, A.; Matyjaszewski, K., Thermodynamic Components of the Atom Transfer Radical Polymerization Equilibrium: Quantifying Solvent Effects. Macromolecules 2009, 42, 6348-6360.

8. Horn, M.; Matyjaszewski, K., Solvent Effects on the Activation Rate Constant in Atom Transfer Radical Polymerization. Macromolecules 2013, 46, 3350-3357. 